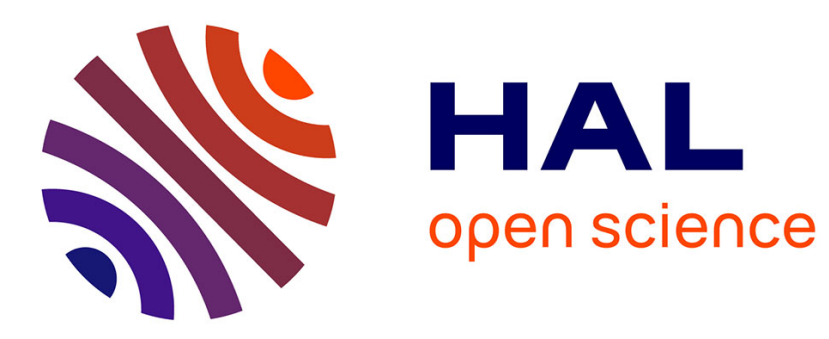

\title{
Interopérabilité des entreprises. Vers l'utilisation d'ontologies éphémères
}

Gregory Zacharewicz, Bruno Vallespir, David Chen

\section{To cite this version:}

Gregory Zacharewicz, Bruno Vallespir, David Chen. Interopérabilité des entreprises. Vers l'utilisation d'ontologies éphémères. Revue des Sciences et Technologies de l'Information - Série ISI : Ingénierie des Systèmes d'Information, 2010, 15 (5), pp.11-36. 10.3166/isi.15.5.11-36 . hal-00555604

\section{HAL Id: hal-00555604 https://hal.science/hal-00555604}

Submitted on 8 Aug 2018

HAL is a multi-disciplinary open access archive for the deposit and dissemination of scientific research documents, whether they are published or not. The documents may come from teaching and research institutions in France or abroad, or from public or private research centers.
L'archive ouverte pluridisciplinaire HAL, est destinée au dépôt et à la diffusion de documents scientifiques de niveau recherche, publiés ou non, émanant des établissements d'enseignement et de recherche français ou étrangers, des laboratoires publics ou privés. 


\title{
Interopérabilités des entreprises
}

\section{Vers l'utilisation d'ontologies éphémères}

\author{
Gregory Zacharewicz - Bruno Vallespir - David Chen \\ IMS UMR CNRS 5218/Groupe Productique - Université de Bordeaux \\ 351, cours de la Libération \\ F-33405 Talence cedex \\ \{gregory.zacharewicz, bruno.vallespir,david.chen\}@ims-bordeaux.fr
}

RÉSUMÉ. Cet article propose, premièrement, une revue des recherches en cours dans le domaine de l'interopérabilité d'entreprise. Une focalisation est ensuite opérée sur les travaux récents portant sur le concept d'interopérabilité d'entreprise selon l'approche "entreprises fédérées » et utilisant la notion émergente d' "ontologie éphémère ». Un rappel des notions de simulation distribuée, du standard HLA et des ontologies permet d'éclairer les concepts qui viendront supporter la mise en æuvre de ces travaux. Leur complémentarité est démontrée pour construire une plateforme logicielle Agent/HLA, basée sur l'usage d'ontologies éphémères, afin de permettre l'interopérabilité d'une nouvelle génération de systèmes d'information agiles. En détail, les ontologies permettent l'échange et la réconciliation des informations "en ligne", point fondamental de l'approche fédérée. Au niveau implémentation, le standard HLA, initialement dédié à la simulation distribuée, est transposé pour assurer l'interopérabilité des systèmes d'information d'entreprises, en réutilisant l'expérience acquise en systèmes distribués. Enfin, une étude compare un ensemble de logiciels, facilitant l'interopérabilité d'entreprises, avec celui spécifié dans cet article. Des limitations sont identifiées et des perspectives sont proposées.

ABSTRACT. This article is proposing first a review of ongoing researches in Enterprise Interoperability. Then, it focuses on the Federation oriented Enterprise Interoperability concept, by means of the rising notion of Short-Lived Ontology. A recall on distributed simulation, HLA standard and short lived ontology exposes their pertinent combination to support execution of the studied concept. Indeed, on the one hand Ontology fits properly the concept of "online" information interoperability matching in a federated enterprise interoperability approach. On the other hand, the HLA standard, initially designed for distributed $M \& S$ purpose, can be transposed for enterprise interoperability at the implementation level, reusing the years of experiences in distributed systems. From these postulates, we propose the first short-lived ontology based Agent/HLA framework to implement distributed enterprise information systems. Finally, a comparison study between some enterprise interoperability software solutions and the one introduced in this article is proposed. From this comparison, limitations are identified and opportunities are given.

MOTS-CLÉS : interopérabilité d'entreprises, système d'information, HLA, ontologies.

KEYWORDS: enterprise interoperability, information system, HLA, ontology.

DOI:10.3166/ISI.15.5.11-36 @ 2010 Lavoisier, Paris

RSTI - ISI - 15/2010. Modélisation d'entreprise, pages 11 à 36 


\section{Introduction}

Dans le contexte économique globalisé, la compétitivité d'une entreprise dépend non seulement de sa productivité interne et de son rendement, mais aussi de sa capacité à collaborer avec ses partenaires. Cette nécessité a conduit à l'élaboration d'un nouveau concept dénommé «interopérabilité », qui tend à améliorer les collaborations entre les entreprises. Nul doute, dans le contexte où les compagnies sont définies par des réseaux d'entreprises partenaires de plus en plus denses et complexes, que l'interopérabilité d'entreprise soit considérée comme une alternative à l'intégration totale des entreprises dans un cadre de fonctionnement unique et irréversible. Depuis le début des années 2000, plusieurs projets de recherche, notamment européens, ont été lancés pour développer l'interopérabilité des entreprises (IDEAS, ATHENA, INTEROP). Trois principaux thèmes ou domaines de recherche qui traitent des questions d'interopérabilité ont été identifiés : 1) la modélisation d'entreprise (ME) qui traite de la représentation des entreprises interconnectées visant à établir des exigences d'interopérabilité ;2) les architectures et plateformes $(\mathrm{A} \& \mathrm{P})$ qui définissent des solutions de mises en œuvres pour réaliser l'interopérabilité ; 3) les ontologies (ON) portant sur la sémantique nécessaire pour assurer l'interopérabilité.

Dans cet article, le concept d'entreprises distribuées souhaitant interagir en tant que fédérés interopérables (entités distribuées conservant leur autonomie) est retenu. Une spécification de mise en œuvre de ce concept est proposée par une plateforme collaborative assurant la communication des systèmes d'information d'entreprises fédérées. Il sera fait appel à la norme d'exécution distribuée (HLA) et aux agents logiciels utilisant des ontologies éphémères pour interpréter les informations échangées. Les ontologies éphémères permettent de s'adapter au caractère très évolutif de ces interactions.

Dans la section 2, les différentes dimensions de l'interopérabilité d'entreprise et la conclusion donnée par le Réseau d'EXcellence INTEROP (PC6, 508011) (Chen, 2007) sont rappelées. Ensuite, les concepts de simulation distribuée et la norme d'exécution/simulation distribuée High Level Architecture (HLA) sont évoqués. Puis une nouvelle catégorie d'ontologies est introduite, permettant de réconcilier sémantiquement, dynamiquement et éphémèrement des informations. Sur la base de ces concepts, une nouvelle plateforme d'interopérabilité est proposée. Enfin, les caractéristiques de la plateforme proposée sont comparées à des plateformes collaboratives existantes afin de révéler les atouts et les limitations de ces plateformes au regard des concepts d'interopérabilité.

\section{Problématique}

Une information possède une structuration et une sémantique qui nécessitent d'être maitrisées pour pouvoir l'utiliser. Le défi scientifique des travaux présentés 
dans cet article est de donner les clés pour rendre l'information intelligible par tous, ou du moins par ceux qui y sont autorisés.

Les entreprises échangent des informations qui augmentent en volume et dont la structure et la sémantique évoluent régulièrement. Les outils logiciels des systèmes d'information (SI) doivent devenir agiles pour réagir à ces besoins d'interopérabilité. A partir de cette problématique industrielle exprimée, cet article propose d'étudier et de solutionner trois aspects de l'interopérabilité essentiels à ces SI collaboratifs.

La première préoccupation d'interopérabilité d'entreprise retenue est la gestion de la temporalité des informations. L'aspect dynamique doit être abordé avec des techniques fiables. Le deuxième aspect concerne la caractérisation sémantique des données d'entreprises. En effet, l'interopérabilité fédérée nécessite une nouvelle approche pour la manipulation et l'interprétation qualitative des données échangées dans un contexte très évolutif. Le dernier aspect concerne la confidentialité des données. Les entreprises doivent gérer la sécurité des données échangées entre entités. Des niveaux de droits sur les données d'entreprises doivent, en conséquence, être définis et gérés. Les ontologies informatiques et le langage des agents logiciels peuvent donner des clés aux considérations sémantiques et causales. En outre, l'expérience des SI, de la modélisation et simulation (M\&S) et du génie logiciel définissent des règles pour synchroniser et sécuriser les données échangées.

Dans cet article, des solutions sont proposées dans ce nouveau contexte d'entreprises fédérées. Au niveau implémentation, la combinaison du dialogue d'agents logiciels distribués, employant des ontologies éphémères, et des mécanismes de simulation distribuée permettra d'établir la communication entre les SI d'entreprises.

\section{Présentations des concepts et des moyens mis en ouvre}

\subsection{Concepts basiques d'interopérabilité}

L'interopérabilité d'enterprise désigne la capacité d'interaction entre systèmes d'entreprises. L'interopérabilité est considérée comme significative si les interactions peuvent avoir lieu à plusieurs niveaux d'abstractions pour un problème donné, dans un contexte professionnel défini et avec une sémantique définie. L'objectif est de s'attaquer aux problèmes d'interopérabilité à travers l'identification des barrières (incompatibilités) qui bloquent l'interopérabilité des systèmes. Les concepts de base relatifs à l'interopérabilité des entreprises peuvent être classés selon trois grandes dimensions (Chen et al., 2007) représentées graphiquement par la figure 1 et décrites ci-après. 


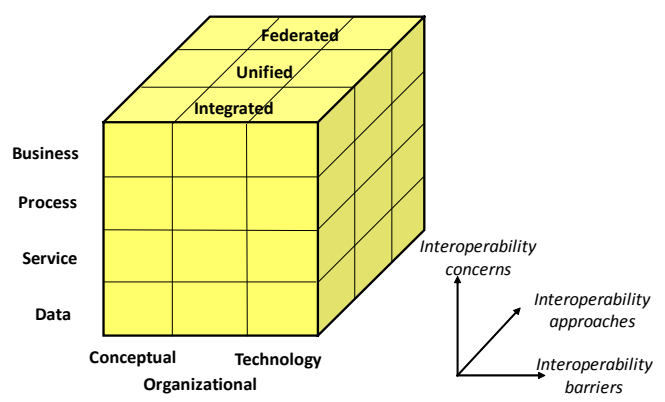

Figure 1. Cadre de l'interopérabilité d'enterprise

\subsubsection{Dimension : barrières d'interopérabilité}

Cette dimension considère trois catégories de problèmes qui peuvent prévenir l'interopérabilité (Ullberg et al., 2009) :

- les barrières conceptuelles sont liées à des problèmes syntaxiques et sémantiques des informations à échanger. Cette catégorie d'obstacles concerne la modélisation à haut niveau d'abstraction, ainsi que le niveau de codage ;

- les barrières organisationnelles sont liées à la définition des responsabilités et de l'autorité pour que l'interopérabilité puisse avoir lieu dans de bonnes conditions ;

- les barrières technologiques sont liées au problème de l'utilisation des technologies de l'information. Cette catégorie d'obstacles concerne les normes qui sont utilisées pour présenter, stocker, échanger, traiter et communiquer des données à travers l'utilisation de moyens informatiques.

\subsubsection{Dimension : niveaux de préoccupation de l'interopérabilité}

Cette dimension identifie les différents niveaux décisionnels et opérationnels de l'entreprise où l'interopérabilité doit opérer. Bien que la catégorisation suivante soit principalement issue de l'architecture ATHENA (Berre, 2007) dont l'objectif est la définition d'un système d'information, elle peut être généralisée à des systèmes non informatiques.

- Le niveau business réfère à l'harmonisation au niveau décisionnel et stratégique de l'organisation du travail de l'entreprise. Cette harmonisation doit surmonter la coexistence de différents processus décisionnels, méthodes de travail, législations, cultures d'entreprise et approches commerciales, etc., coexistant au sein des filiales de la société.

- Le niveau processus vise à faire travailler ensemble différents processus. Un processus définit une séquence de services ou de fonctions permettant d'assurer un besoin spécifique d'une société considérée. Généralement, dans une entreprise, plusieurs processus s'exécutent en série, parallèle et en interaction. Dans le cas d'une 
entreprise virtuelle en réseau de sous-traitants, les processus internes des soussociétés doivent être connectés à leur environnement (à l'extérieur) pour créer le processus global commun.

- Le niveau service est préoccupé par l'identification, la composition, et le fonctionnement de différentes applications (conçues et mises en œuvre indépendamment) en résolvant les différences syntaxiques et sémantiques, ainsi que les connexions à trouver entre diverses bases de données hétérogènes. Le terme «service» n'est pas limité aux applications informatiques, mais également aux tâches non automatiques.

- Le niveau données se réfère à l'implémentation des différents modèles de données (hiérarchiques, relationnels, etc.) et aux différents langages de requêtes qui les manipulent. L'interopérabilité des données consiste à identifier et partager des informations provenant de bases hétérogènes, qui peuvent résider sur différents dispositifs physiques avec des systèmes d'exploitation, des langages informatiques et des systèmes de gestion de bases de données hétéroclites.

\subsubsection{Dimension : approches d'interopérabilité}

Cette dimension prend en considération les trois approches employées pour développer l'interopérabilité. Elles sont présentées ci-après chronologiquement dans l'histoire récente de l'interopérabilité.

- Approche intégrée. Les entreprises reconnaissent un format commun pour tous les modèles employés et les réalisations engendrées. Ce format doit être aussi détaillé que les modèles. Le format commun n'est pas nécessairement une norme mais doit être accepté par toutes les parties élaborant les modèles et construisant les systèmes.

- Approche unifiée. Les entreprises utilisent leurs propres formalismes ou standards. Cependant, il existe un format commun aux interfaces des entreprises (seulement à un métaniveau). Ce métamodèle n'est pas une entité exécutable comme il l'est dans l'approche intégrée, mais il fournit un moyen d'équivalence sémantique pour permettre la transposition des concepts entre les modèles. Une norme de correspondance (pivot) doit être proposée. Par exemple dans le domaine de la simulation distribuée, il peut être cité CORBA, ALSP, DIS, HLA (Fujimoto, 2002). Ces normes aident les systèmes hétérogènes à communiquer sans recodage des systèmes réutilisés dans le langage de l'un d'entre eux.

- Approche fédérée. Les entreprises ne possèdent pas de formalisme commun. Les parties doivent s'accommoder à la volée. Cette approche «fédérée » implique qu'aucun partenaire n'impose ses modèles, langages et méthodes de travail. Les entreprises doivent pouvoir interpréter l'information reçue à partir de leurs expériences et de leurs connaissances antérieures sur les structures de représentation des données dans le(s) domaine(s) où elles travaillent. L'utilisation d'ontologies de domaine, jointes aux informations transmises ou bâties localement par expérience, peut contribuer à cette compréhension. 
La plupart des approches d'interopérabilité développées actuellement sont unifiées. Par exemple dans le domaine de la modélisation d'entreprise, les formalismes Unified Enterprise Modeling Language (UEML, 2003) et Process Specification Language (PSL, 2003) visent à soutenir l'interopérabilité entre modèles d'entreprise par des formalismes unifiant la représentation des concepts.

La recherche et la mise en œuvre sur le terrain de l'approche fédérée pour développer l'interopérabilité d'entreprises est la motivation des recherches actuelles des auteurs. Ces travaux sont guidés en cela par les besoins des entreprises actuelles qui évoluent dans un contexte de plus en plus dynamique, se traduisant par des collaborations à durées déterminées. En détail, l'approche fédérée aura pour objectif de développer une interopérabilité maximale de l'entreprise avec son environnement et sera particulièrement adaptée à un cadre inter-organisationnel (entreprises en réseau, entreprises virtuelles, etc.). La feuille de route pour l'interopérabilité des entreprises publiée par la Commission Européenne (IST, 2007), présente l'approche fédérée pour l'interopérabilité ( $3^{\mathrm{e}}$ génération) comme l'un des défis de la recherche pour les années à venir. Néanmoins, peu d'activités de recherche ont été effectuées dans cette direction (IST, 2007).

\subsection{Simulation distribuée}

Le but de la simulation distribuée est d'optimiser l'utilisation des ressources, de travailler sur des ressources distantes et/ou de réutiliser des simulations existantes, et plus généralement des systèmes, en les interconnectant. Un traitement distribué doit garantir la confidentialité, l'intégrité et les relations de causalité temporelles en utilisant des algorithmes de synchronisation.

La simulation distribuée est retenue pour assurer l'échange d'informations entre les SI car elle permet de traiter des données hétérogènes provenant de systèmes distribués également hétérogènes et ceci sans les interpréter. De plus, elle possède des mécanismes permettant l'échange de messages synchronisés. Ce n'est qu'un moyen de véhiculer et d'orchestrer l'échange des données entre SI, « alternatif » au SOA, robuste (exécuté à bas niveau, exprimé par des modèles à événements discret, type DEVS (Zeigler, 1973)) et complètement explicite (algorithmes de synchronisation ; Fujimoto, 2000). L'exécution (ou la simulation) locale et l'interprétation des messages échangés restent à la charge de la définition du comportement du SI local et ne sont pas abordées dans cet article.

\subsubsection{Norme et concepts HLA}

L'architecture de haut niveau High Level Architecture (HLA) est une spécification qui définit une architecture logicielle pour créer une exécution logicielle globale composée de simulations et d'applications distribuées. Cette norme a été introduite pour la modélisation et la simulation du Defense M\&S Office (DMSO) de l'US Department of Defense (DOD). L'objectif initial était la 
réutilisation et l'interopérabilité des applications militaires, des simulations et de capteurs/actionneurs en prise avec un environnement réel.

Dans la norme HLA, toute application participante est appelée fédéré. Les fédérés interagissent au sein d'une fédération HLA (groupe de fédérés). L'ensemble de définitions HLA a suscité la création d'une norme notée 1.3 en 1996, qui a évolué vers HLA 1516, en 2000 (IEEE, 2000).

La spécification des interfaces des entités HLA décrit la communication au sein de la fédération et définit la mise en œuvre d'un composant de coordination : le Run Time Infrastructure (RTI). Les fédérés interagissent avec les services proposés par la RTI. Ils peuvent notamment «Publier» pour informer de leur intention d'envoyer des informations à la fédération et s'« Abonner » pour tenir compte des informations créées et mises à jour par les autres fédérés. Les informations échangées dans HLA sont représentées sous la forme de classes d'objets au format XML. Les services d'échange d'informations distribuées du RTI HLA sont détaillés dans (IEEE, 2000).

Dans le contexte d'interopérabilité d'entreprise, HLA semble être une solution appropriée pour établir l'interopérabilité entre les systèmes d'information des entreprises. HLA gère l'hétérogénéité des systèmes et met en place un «système de systèmes » au lieu d'une solution monolithique. Chaque système d'entreprise est un fédéré HLA qui est synchronisé avec les autres par le RTI HLA. La solution n'utilise en fait que la partie distribution/synchronisation de HLA et non la partie simulation comportementale. La simulation distribuée est utilisée comme une alternative aux SOA plus robustes au regard de la gestion de l'intégrité causale des messages.

\subsubsection{Simulation distribuée basée agent}

Le système multi-agent (SMA) se concentre sur l'étude du comportement collectif, qui résulte de l'organisation, et des interactions d'Agents pour la résolution de problèmes. Un SMA est un système distribué dans lequel il n'existe généralement pas de contrôle centralisé et de point de vue global. Un SMA est composé d'agents qui agissent de façon autonome, mais qui ne possèdent pas localement toutes les compétences, les ressources ou les informations requises pour assurer la cohérence globale d'une action concertée. Les agents perçoivent les modifications de leur environnement et y réagissent. Parmi les actions possibles, les agents doivent déterminer les décisions appropriées pour atteindre leurs objectifs. Le domaine d'application, l'environnement et le type d'interaction influencent la spécification de la conception des agents. De nombreuses applications basées SMA sont aujourd'hui développées. Wooldridge (2002) propose une revue de l'utilisation d'agents en fonction de différents domaines d'application (agents cognitifs, agents logiciels, agents mobiles, etc.). 


\subsection{Concept d'ontologie éphémère}

Selon (Gruber, 1993), en informatique et en sciences de l'information, une ontologie est une représentation formelle d'un ensemble de concepts d'un domaine qui de plus explicite les relations entre ces concepts. Elle est utilisée pour raisonner sur les propriétés de ce domaine, et peut être utilisée pour définir le domaine. L'ontologie de domaine est une structuration d'un aspect précis ou d'une facette du monde (un champ de travail, un métier, etc.) ou, du moins, la représentation humaine de celui-ci.

Deux classes d'ontologies peuvent être différenciées. Les ontologies techniques et les ontologies métiers. Les premières sont dédiées à la définition des éléments et des moyens mis en œuvre pour l'implémentation (bas niveau). Ces ontologies considèrent par exemple les protocoles de communication pour l'échange d'informations. La deuxième classe est assimilée à l'ontologie de domaine qui décrit les concepts et leurs relations au sein d'un métier. Les ontologies éphémères sont celles de domaine. Fondamentalement, il est possible d'ajouter une valeur sémantique sur le lien entre les concepts. Cette approche, décrite notamment dans (Gandon, 2008), est basée sur le modèle entités relations des données informatiques. Le niveau de proximité est défini en ajoutant une pondération sur les liens telle que décrit par (Rada, 1989) et plus récemment (Zhong, 2002). Ainsi, la collecte, l'analyse et l'interprétation d'informations peuvent être automatisées et facilitées par l'utilisation d'une approche ontologique.

a.

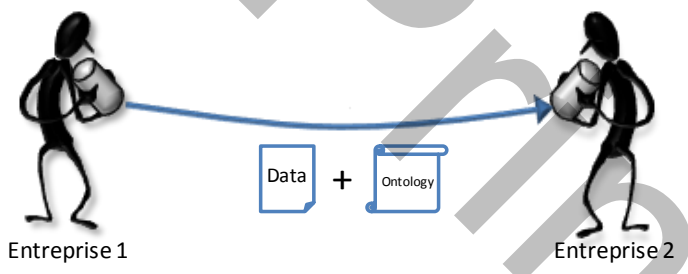

b.

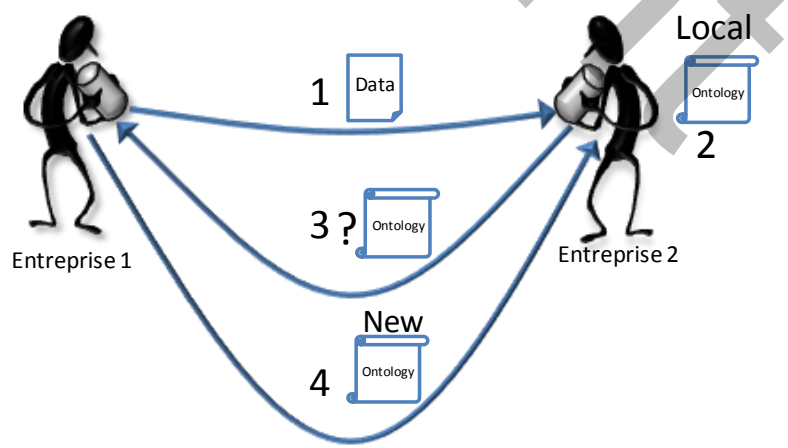

Figure 2. Echange de données en interopérabilité fédérée 
Cependant, dans l'approche d'interopérabilité fédérée d'entreprises, aucune persistance de metamodèle commun n'est supposée exister; le participant doit interpréter la communication à la volée. En conséquence, Zacharewicz et al. (2009) ont estimé que les structures des messages échangés et leurs ontologies doivent être de courtes durées (non persistantes). Dans la suite, ce mécanisme de communication est illustré informellement où deux cas peuvent être distingués (figure 2).

- Cas a : l'entreprise 1 envoie une information et une ontologie pour interpréter le sens de l'information (décodage) simultanément. Cette ontologie n'est supposée valable que pour cette information. L'ontologie n'est donc pas persistante dans les échanges des deux entreprises.

- Cas $b:$ l'entreprise 1 envoie uniquement une information vers l'entreprise 2. Une fois que l'entreprise 2, reçoit l'information, elle interprète la signification à l'aide de sa base d'ontologies locales et détermine si elle est capable de décoder les informations. Sans quoi, elle demande à l'expéditeur l'ontologie associée au message. L'entreprise peut conserver l'ontologie reçue et la réutiliser pour d'autres données envoyées par cet émetteur ou un autre se conformant à la même ontologie. Une date de validité peut être associée à l'ontologie.

Dans la première solution, les informations peuvent être exploitées directement grâce à l'ontologie reçue simultanément. Toutefois, la taille des informations échangées est plus importante. En outre dans cet échange systématique, l'ontologie peut être interceptée et la confidentialité peut être rompue. Dans le second cas, la confidentialité est accrue, mais davantage d'échanges entre les deux partenaires pour trouver le sens du message peuvent être requis, par conséquent la durée de la communication augmente. Les besoins de confidentialité et de performance ont conduit à focaliser l'étude sur la deuxième solution. Ce postulat a permis de créer la notion d'ontologie « courte durée » ou «éphémère ». Cette définition d'ontologie est basée sur une spécialisation de la définition de Gruber (1995). Il est ajouté à cette définition que l'ontologie peut être, dans certains cas, supprimée après utilisation et qu'elle possède une validité de durée finie. Cette solution respecte ainsi mieux l'exigence d'interopérabilité « à la volée » de l'approche fédérée.

La question sous-jacente à l'approche fédérée en interopérabilité consiste à savoir s'il n'existe pas toujours, à un metaniveau, une référence ou un standard pour pouvoir communiquer. La réponse est affirmative mais cette «ontologie » persistante est pauvre sémantiquement, elle définit simplement la syntaxe des protocoles d'échange de messages ou d'information. Cette référence est une «ontologie technique » qui se situe dans les niveaux bas (service et données) de la dimension conceptuelle de l'interopérabilité. Les entreprises ne partagent donc pas les concepts au niveau business de façon permanente. Par exemple, cette ontologie permet de déterminer que l'information est une «chaîne de caractères », un «mot» (par opposition à une valeur numérique par exemple) contenant des caractères alphabétiques. Mais cette ontologie ne donne aucun sens particulier à ce mot. 


\section{Systèmes d'information interopérables}

\subsection{Points d'interopérabilité à assurer}

La première partie de la section précédente a présenté les concepts d'interopérabilité souhaitables pour les entreprises. Puis les concepts de systèmes interopérables utilisant HLA et Agents ont été rappelés. Cette section identifie plusieurs barrières d'interopérabilité que l'étude présentée dans cet article propose d'éliminer par l'implémentation d'Agents et des concepts HLA.

\subsubsection{Réconciliations syntaxiques et sémantiques par ontologie}

Les concepts manipulés, les structures organisationnelles adoptées et les technologies employées dans une entreprise peuvent n'avoir aucune signification équivalente dans une autre. Cependant, dans le cadre d'une coopération, les entreprises ont besoin d'un rapprochement (ou d'une mise en correspondance) des concepts qu'elles manipulent respectivement, de leurs structurations organisationnelles et de leurs technologies pour interpréter correctement les informations qu'elles veulent échanger.

Les ontologies de domaine, présentées dans la section précédente, peuvent aider à réconcilier les discordances de concepts (i.e. des barrières conceptuelles) exprimées dans les différentes entreprises impliquées dans un échange d'information. Ce principe repose sur une référence sémantique à des metaconcepts communément reconnus par l'ensemble des entreprises. Cependant, dans l'approche d'interopérabilité fédérée, les mappages sémantiques des informations échangées doivent pouvoir se faire en «peer-to-peer » lors de l'exécution, et ce, pour une durée déterminée. Ce contexte nécessite la compréhension des concepts en temps réel. La référence sémantique commune doit donc être transmise, utilisée et admise le temps de la collaboration (éphémère), elle doit pouvoir être collectivement enrichie, mais également supprimée, par les systèmes d'information fédérés. Ainsi, la référence peut être une ontologie qui est transmise (donc partagée) entre des entreprises pour une durée finie. Cette définition d'ontologie présentée en section 3.3 a été introduite sous le terme ontologie éphémère (ou de courte durée) par (Zacharewicz, 2009).

En outre, lorsque les structures organisationnelles diffèrent entre entreprises qui souhaitent coopérer (i.e. présence de barrières organisationnelles), des dysfonctionnements peuvent se produire. Par exemple, dans une relation client/fournisseur ou dans le cadre d'un projet commun, une ressource émettrice d'une entreprise peut rencontrer des problèmes pour identifier l'interlocuteur adéquat qui pourra recevoir l'information dans l'entreprise partenaire. Ces problèmes sont relatifs à la description uniquement syntaxique de la hiérarchie de l'organisation des entreprises. Les informations échangées comportent des propriétés sémantiques implicites qui peuvent permettre d'extraire des attributs. Le traitement sémantique de l'information, en utilisant une ontologie, peut faciliter, par exemple, la détermination des destinataires d'une information. Au niveau du système 
d'information les attributs révélés permettent d'identifier par leurs profils informatiques (ex. login, adresse mail, etc.) un acteur destinataire (humain ou logiciel) capable de traiter l'information automatiquement.

Au niveau technologique, un inventaire des ontologies d'entreprise (Chen et al., 2003) fait apparaître la prépondérance du format XML pour porter les informations entre systèmes d'information fédérés (et franchir des barrières technologiques). Plus généralement, les principaux corpus d'ontologies sont aussi mis en œuvre en XML (RDF/S, DAML et OWL). De plus, XML permet la définition de DTD (Data Type Definition) ou de schéma XML dont le but est de représenter et structurer les données dans un langage balisé standardisé (supprimant des barrières technologiques liées à l'utilisation de langages hétérogènes). Les structures de données sont des grammaires qui vérifient la cohérence d'un document XML. L'ontologie peut être construite sur ces grammaires. Les considérations énoncées dans cette section sont relatives à la description statique des systèmes d'information.

Par ailleurs, le processus de collaboration qui définit l'échange des informations entre entreprises doit être formalisé. Un modèle de flux de tâches (Workflow) permet de représenter les séquences d'échange des SI. La modélisation de Workflow (ou BPM) représente le processus de traitement de produits physiques ou de données. Elle décrit les séquences d'échange d'information entre partenaires et définit les rôles des acteurs des entreprises. La WfMC (Workflow Management Coalition) a choisi XML comme représentation de la norme Workflow (WFMC 2005). La gestion d'un tel processus fait appel à un moteur d'orchestration dont le mécanisme est dirigé par les événements à traiter. Le format XML est également utilisé par la norme HLA qui définit les objets et l'interaction dans ce format commun. Ces données définies selon la structure classique de programmation objet permettent de faciliter la constitution et la réutilisation de bibliothèques de données partagées (persistantes ou non) entre systèmes d'information hétérogènes répartis.

Enfin, la possibilité de transformer la structure des données XML par l'utilisation d'un processeur XSL (Extensible Stylesheet Language) apporte cohérence et robustesse. Les données peuvent être transformées verticalement pour atteindre un niveau d'abstraction souhaité et horizontalement entre formalismes différents. Ceci en définissant un mécanisme (autant que possible) automatisé de transformation et d'enrichissement (ex. par référence à une ontologie validée par des experts du domaine), tel que présenté dans le projet LSIS-WME (Zacharewicz et al., 2008).

\subsubsection{Causalité temporelle dans les systèmes d'information d'entreprises}

L'approche fédérée pousse plus loin la notion d'interopérabilité d'entreprise. Au cours de l'exécution, l'entreprise a besoin d'échanger des messages à la volée, tout en conservant un comportement déterminé par les spécifications du modèle d'entreprise. Dans ce contexte, une préoccupation consiste à surmonter des obstacles organisationnels et technologiques pour être sûr de respecter la causalité temporelle et la chaîne de causalité des actes sur l'information. En outre, dans l'approche 
d'interopérabilité fédérée, les droits sur l'information peuvent évoluer. Après une période de coopération dans un projet commun, ce droit peut être modifié en fonction de la nouvelle position de l'entreprise (par exemple, responsable de lot de travail dans un projet, participant à un lot, ne participant plus au projet, etc.). Ce sont clairement des barrières organisationnelles. Par ailleurs, l'identification et l'expression du problème de causalité dans le modèle de processus (barrières conceptuelles) facilitent l'implémentation (barrières technologiques).

Pour conforter ce propos, Chen et al. (2007) résument les recherches actuelles et identifient de la même façon la nécessité d'une gestion de l'espace mais aussi du temps pour éliminer au mieux les barrières conceptuelles. La modélisation et l'approche simulation distribuée proposent aujourd'hui des solutions matures. En particulier, le respect de la causalité temporelle de l'information échangée est traité depuis la fin des années 1970 avec l'introduction d'algorithmes de synchronisation (Fujmoto, 2000). Plus tard, les normes (IEEE, 2001), par exemple, impliquent ces algorithmes pour soutenir et faciliter le développement de plateformes distribuées pour des applications de modélisation et simulation. Par exemple, dans (Zacharewicz et al., 2006), l'interaction entre des modèles DEVS (Zeigler, 1973) distribués est détaillée. Par conséquent, la synchronisation des modèles d'entreprise peut considérer l'expérience de ce domaine. Concrètement, la simulation énonce des règles connexes au temps (i.e. à la causalité), ces règles peuvent être utilisées pour régir le comportement du système d'information distribué.

\subsubsection{Gestion du partage des données (confidentialité des informations)}

Dans une relation de partenariat, de sous-traitance ou de client/fournisseur entre entreprises, il est nécessaire de définir des responsabilités parmi les ressources (humaines ou logicielles) des entreprises, et notamment le droit d'émettre, de recevoir et de traiter l'information. C'est un problème qui doit être clairement pris en compte (barrière organisationnelle). Ces propriétés et droits concédés aux ressources peuvent évoluer en cours d'exécution et doivent être intégrés dans le modèle de processus de collaboration qui définit la relation entre entreprises (barrière conceptuelle). Concernant toujours ce postulat, l'autre principal problème d'interopérabilité est la gestion de la confidentialité des données au niveau implémentation (barrière technologique).

La norme (ISO, 1999) statue sur l'obligation pour une entreprise de partager certaines données sélectionnées dans un objectif d'interopérabilité, mais prescrit de maintenir les autres informations de l'entreprise confidentielles, c'est la définition de données privées publiques. La norme HLA, réalisée initialement dans l'objectif d'échange d'information entre simulation distribuée militaire, permet de solutionner ce problème. HLA fonde son approche sur la théorie des ensembles : elle définit des sous-ensembles de données à partager par interopérabilité et d'autres sous-ensembles qui ne doivent pas être révélés aux composants extérieurs. Plus en détails, les données de l'entreprise doivent être partitionnées en ensembles classés avec des niveaux de confidentialité. Ce champ est bien développé dans les jeux de guerre 
militaires interopérables (IEEE, 2001) et peut être transposé à la problématique des entreprises fédérées.

Dans le détail, HLA emploie la théorie des ensembles mathématiques pour définir des sous-ensembles de données pouvant être échangées ou rester confidentielles. En fonction de ses droits, le fédéré peut partager, mettre à jour, manipuler et recevoir des données. Ces définitions peuvent être généralisées pour la définition des relations entre systèmes d'information d'entreprises distribuées.

\subsection{Systèmes d'information collaboratifs interopérables}

Les besoins croissants d'interconnexion des entreprises, font naître une nouvelle catégorie de logiciels pour l'échange d'informations dans les années 1990. Les plateformes de collaboration, décrites par (Timmers, 1998), sont des plateformes logicielles (d'interopérabilités unifiées) qui facilitent la communication synchrone et asynchrone grâce à une variété de services et de canaux de communication.

\subsubsection{Premiers systèmes d'information collaboratifs interopérables}

En 1999, Leigh et al. présentent CAVERN, un système distribué pionnier pour l'échange d'informations entre partenaires distribués. Dans cette approche, les obstacles technologiques sont au centre de la considération. Cette approche se situe, dans le cadre d'interopérabilité de l'entreprise (figure 1), aux niveaux « service et données », avec une approche "intégrée et unifiée » (figure 3). Ce système vise à définir une interface permettant de connecter le système avec d'autres systèmes hétérogènes. Ce système est unifié dans le format de l'échange d'informations mais reste finalement intégré dans sa mise en œuvre. Les concepteurs définissent des profils d'accès à l'information, mais l'élaboration de services composables n'est pas proposée. En outre, il ne définit pas la séquence d'actions sur les données (le processus). Les accès à l'information peuvent être concurrents et simultanés.

Panetto et al. (2004) ont présenté une revue des plateformes existantes. Ils décrivent de nombreux projets de plateformes collaboratives et situent la plateforme INTEROP qu'ils proposent. Leurs travaux intègrent l'idée que le développement de la plateforme commence à partir d'un modèle conceptuel d'entreprise (au niveau des notions métiers des entreprises considérées). En conséquence, ils élargissent le champ de portée de l'étude et intègrent une vision de plus haut niveau de l'interopérabilité. Ils formalisent les concepts, aidant ainsi la compréhension des fonctions et du comportement de la plateforme.

La figure 3 présente les approches et plateformes CAVERN et INTEROP qui sont projetées sur le cadre d'interopérabilité afin d'observer leur couverture fonctionnelle. Les deux plateformes permettent de définir un service dédié aux profils des utilisateurs et dépendant du droit d'accès aux données. Le problème technique est également bien adressé au niveau d'abstraction le plus bas 
(implémentation). Néanmoins, dans ces deux plateformes, les responsabilités organisationnelles ne sont abordées que sur le plan droit d'accès aux données. Il est également possible de distinguer que seule la deuxième plateforme intègre clairement l'identification et la modélisation des concepts au niveau business avant de les mettre en œuvre dans un contexte technologique particulier.
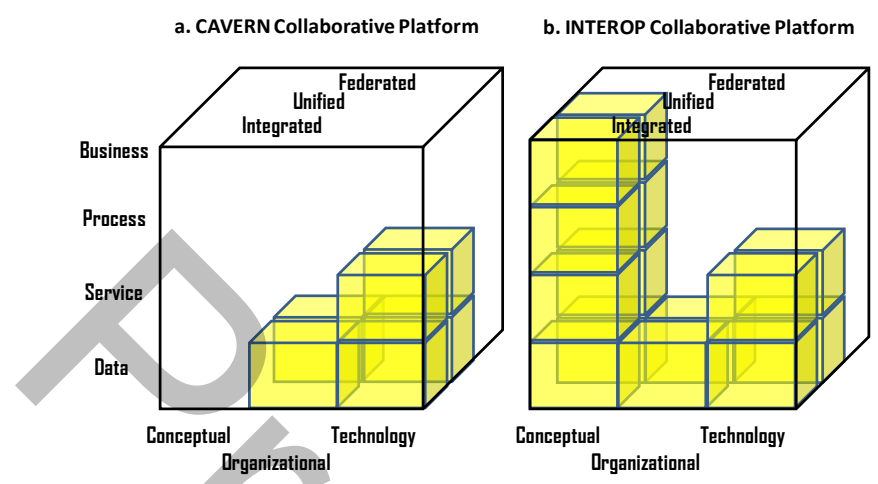

Figure 3. Couverture du cadre d'interopérabilité d'entreprise

Depuis la fin des années 1990, il est possible d'observer que la plupart des approches développées sont passées d'une approche intégrée vers unifiée comme indiqué par la figure 3. Pour faciliter cette approche non intégrée, l'entreprise a besoin de références, normes ou standards pour s'accorder sur l'échange d'informations. Des efforts de formalisation et de modélisation des entreprises ont été effectués dans ce sens. Par exemple dans le domaine de la modélisation d'entreprise, il peut être cité UEML (Unified Enterprise Modelling Language) et PSL (Process Specification Language) qui sont des techniques de modélisation et des langages, et qui visent à soutenir l'interopérabilité entre les modèles d'entreprises et les outils d'implémentation.

L'étude bibliographique menée a mis en évidence, que dans les années 1990 peu d'activités de recherche ont été réalisées au sujet de l'approche fédérée pour développer l'interopérabilité des entreprises. À cette époque, cette approche n'était pas pleinement identifiée, de plus à ce moment, aucune solution logicielle et matérielle n'était suffisamment avancée pour soutenir cette approche. Aujourd'hui, l'approche fédérée, qui vise à développer une forme d'interopérabilité plus flexible (et même agile), est particulièrement attrayante pour les environnements interorganisationnels (comme les entreprises en réseau, les entreprises virtuelles, etc.). La feuille de route sur l'interopérabilité des entreprises publiée par la Commission européenne (IST, 2006) confirme que l'élaboration de solutions et de systèmes d'information par l'approche fédérée pour l'interopérabilité est considérée comme l'un des principaux défis de recherche de ce domaine pour les années à venir. 


\subsubsection{Perspectives pour l'interopérabilité depuis les premières solutions}

L'état de l'art sur l'interopérabilité des entreprises et les deux premières expériences de mises en œuvre à la fin des années 1990, présentées figure 3, indiquent les zones d'interopérabilité qui n'ont pas été abordées par ces premières approches. De ce postulat, plusieurs directions de recherche pour renforcer l'interopérabilité par l'élimination de barrières ont été envisagées au début du $21^{\mathrm{e}}$ siècle.

La première orientation a concerné l'amélioration de la compréhension et de la représentation des réseaux d'entreprises permettant de franchir des barrières conceptuelles. La définition de paradigmes spécifiques pour modéliser les entreprises, type UEML, GRAI (Doumeingts, 1984) (à un niveau conceptuel), ainsi que les langages type UML, Workflow, BPM (à des niveaux d'abstraction plus proches de l'implémentation) ont pu concourir à cette amélioration. Ces formalisations ont permis de rationnaliser la spécification des entreprises afin de mieux comprendre et faciliter la proposition de systèmes d'information, ceci, en s'accordant sur les notions manipulées depuis les concepts jusqu'à la mise en œuvre. Les transformations de ces modèles ont été, quant à elles, encadrées par des méthodologies de type Model Driven Architecture (MDA) (OMG, 2003).

Malgré ce cadrage méthodologies de développement, suite à la phase de formalisation à un niveau conceptuel, il subsiste un certain nombre d'ambiguïtés ou d'imprécisions à lever pour développer un système d'information. En effet ce niveau de modélisation représente l'ensemble d'un système d'entreprise. Dans l'objectif de développer un système d'information il reste à définir la sous-partie de ce système à informatiser. Dans ce sous-ensemble, il apparaît une deuxième exigence non adressée au niveau modélisation d'entreprise : le comportement dynamique, i.e. la synchronisation des informations selon des règles métiers et des responsabilités organisationnelles (des barrières organisationnelles doivent être prises en compte, pour définir les séquences d'accès aux données). Le déroulement approprié des échanges d'informations et la responsabilité des ressources nécessaires pour les traiter sont importantes, l'ignorer peut conduire à des malentendus et au mauvais fonctionnement $\mathrm{du}$ modèle puis du système d'information développé. Le domaine des systèmes d'information traduit l'accès aux données et éventuellement la séquentialité des actions sur les données. Mais la relation aux traitements datés est laissée au domaine de la simulation. La simulation distribuée, en particulier (ex. HLA), considère, de plus, la synchronisation de systèmes distribués et hétérogènes.

Enfin, le travail des entreprises en réseaux de partenaires incite à la sécurisation des données manipulées. La modélisation d'entreprise a dû intégrer la gestion de la confidentialité des données. En particulier, l'interopérabilité peut être considérée entre entreprises partenaires dans un projet mais concurrentes sur d'autres points. Dans ce contexte, une stratégie de partage/protection des données doit définir les responsabilités et les droits des personnes à accéder aux informations dans les 
différentes entreprises du réseau. La sécurisation des applications et des réseaux informatiques a permis de concourir à un contrôle détaillé des informations.

\subsection{Plateformes d'Interopérabilité mises en ouvre en fédérations HLA}

De nombreuses plateformes d'interopérabilité sont apparues depuis les années 1990. Une plateforme d'interopérabilité est un système matériel et logiciel permettant de faire communiquer des systèmes d'information, des applications ou des simulations distants et hétérogènes. Dans le cadre de l'étude développée dans l'article, plusieurs plateformes ont été étudiées. En particulier, il a été observé que les plateformes s'appuyant sur la norme HLA (aujourd'hui employée dans les applications industrielles civiles) résolvent en grande partie les barrières qui subsistaient dans les premières générations de plateformes (voir section 4.2.1). En conséquence, cet article se concentre sur cette catégorie de plateformes. Cette section présente plusieurs approches utilisant HLA pour soutenir la mise en œuvre d'une solution d'interopérabilité au niveau SI d'entreprise. Les trois catégories de barrières à adresser seront observées au regard des fonctionnalités des plateformes. La figure 4 présente un schéma pour chacune des 4 architectures étudiées.

a)

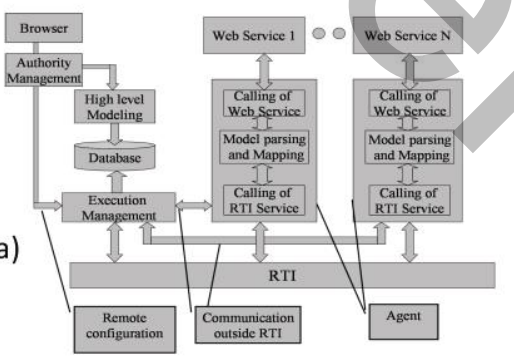

b)

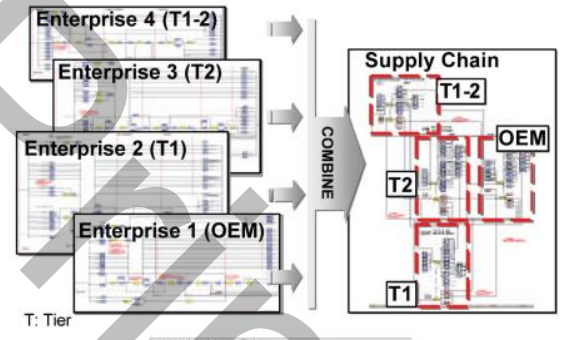

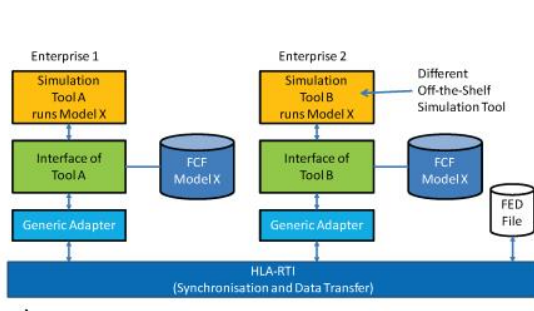

c)

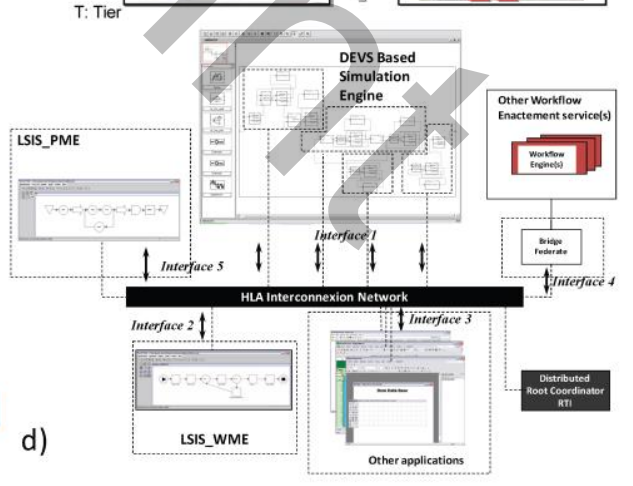

Figure 4. Plateformes d'Interopérabilité HLA 


\subsubsection{Multidisciplinary Collaborative Design of Virtual Prototyping (MCDVP)}

Le développement de produits complexes requiert une connaissance pluridisciplinaire et implique la coopération assistée par ordinateur des concepteurs. Les technologies de la collaboration multidomaine sont des approches efficaces pour la spécification et le développement d'un produit complexe. Zhang et al. (2007) suggèrent d'appliquer les techniques de modélisation \& simulation multidomaine au niveau du développement des produits complexes (figure $4 \mathrm{a}$ ).

L'élément clé de cette approche consiste à intégrer différents outils logiciels autonomes dans un environnement distribué. La nature des composants de cette plateforme de modélisation et simulation pour la conception collaboration multidomaine a nécessité de placer l'interopérabilité au cœur des préoccupations. Dans ce travail, les auteurs proposent une structure fondée sur HLA et les services web pour le développement de solutions d'interopérabilité grâce à une collaboration pluridisciplinaire et dans un contexte d'environnement distribué. Le processus de gestion de projet de la collaboration multidomaine est, en particulier, intégré dans un outil de contrôle centralisé des processus distribué.

\subsubsection{Common enterprise modelling framework's for distributed organisations (CEMF-DO)}

L'objectif de CEMF-DO est de fournir, à des organisations distribuées, une structure commune dans laquelle la modélisation, la simulation, l'analyse et la gestion des modèles pour le développement de solutions d'interopérabilité sont étudiées et intégrées. La mise en œuvre se fait en implantant chaque composant distribué en tant que fédéré HLA (figure 4b) agissant comme une ressource d'un processus considéré.

Les recherches publiées par (Berio et al., 2005) sont liées au langage UEML, ainsi qu'à la gestion et la synchronisation des modèles d'entreprise. Cette recherche a été réalisée pour un lot de travail dans le REx INTEROP (Chen et al., 2007).

\subsubsection{Modelling and Simulation environments for design, planning and operation of globally distributed enterprise (MISSION)}

Les travaux de (Jaekel, 2005) présentent une simulation distribuée de chaînes d'approvisionnement par une approche de type fédération HLA. Dans ce domaine, l'approche proposée par les auteurs se concentre sur la simulation distribuée du processus de distribution et en particulier la chaîne d'approvisionnement. Le concept global est basé sur les résultats du module européen MISSION (Rabe et Jaekel, 2002) et sur une extension de l'IEM (Integrated Enterprise Modelling). Cette approche comprend les aspects de modélisation qui décrivent comment un utilisateur peut collecter les données nécessaires pour la simulation distribuée. En outre, il décrit comment les différents modèles de simulation peuvent être choisis à partir d'une bibliothèque de modèles, puis connectés grâce à un modèle d'entreprise global. L'approche emploie HLA pour soutenir une simulation distribuée d'un 
système industriel. Cette démarche peut être considérée comme une spécialisation de la norme HLA pour les systèmes civils industriels.

Dans cette approche (voir figure 4c), un outil de simulation gère la définition et l'interopérabilité des modèles de simulation par des échanges d'objets. Il propose un outil graphique intuitif pour définir des scénarios de simulation de chaînes de production. L'outil de gestion de simulation garantit la cohérence entre les fichiers de configuration de fédérations pour le scénario de simulation distribuée et génère en conséquence tous les fichiers requis par le RTI pour la simulation.

\subsubsection{LSIS - Workflow model editor (LSIS-WME)}

Dans (Zacharewicz et al., 2008), une plateforme de M\&S «ouverte» est proposée pour créer et exécuter des modèles de flux de production dans le domaine de la fabrication de l'industrie micro-électronique. HLA permet, dans cette plateforme, d'assurer l'interopérabilité de l'environnement de M\&S avec d'autres composants logiciels hétérogènes de l'entreprise, notamment des applications professionnelles spécifiques. La simulation distribuée est utilisée pour simuler une nouvelle chaîne de production, afin d'anticiper de mauvais changements qui pourraient provoquer des dysfonctionnements ou des contre-performances de production.

La figure 4d illustre l'interopérabilité, orchestrée par un HLA RTI, entre l'environnement LSIS-WME et les composants logiciels d'entreprise. Les applications communiquent par publication et abonnement à des informations gérées par la RTI, l'accès aux données est limité par les droits définis de publication/abonnement à des sous-ensembles d'informations. Cette représentation illustre également la capacité de l'environnement à se brancher à d'autres composants hétérogènes et logiciels compatibles HLA.

\section{Implémentation de l'interopérabilité fédérative}

La section précédente fait état du fonctionnement des plateformes collaboratives selon les approches unifiées ou intégrées. Cet article propose une plateforme qui met en œuvre l'approche d'interopérabilité entre entreprises fédérées en utilisant le nouveau concept d'ontologie éphémère. Cette plateforme sera mise en œuvre par des SMA/HLA.

\subsection{Définition de la plateforme}

La proposition part de l'état des besoins d'interopérabilité pour l'interfaçage des entreprises dans le cadre de projets de coopération. Labarthe et al. (2007) présentent une première solution pour établir l'interopérabilité dans la communication des entreprises à l'aide de SMA. Ils proposent de mettre en place un mécanisme de 
communication basé sur des Agents logiciels utilisant la plateforme JADE. Par ailleurs, (Zacharewicz et al., 2008) définissent un moteur Workflow en utilisant des modèles DEVS (Zeigler, 2000) interopérables par HLA. La convergence de ces deux approches présente les bases d'une approche généralisée pour réaliser l'interopérabilité entre composants hétérogènes. L'étude propose, à partir de ces approches, de répondre à deux besoins des entreprises en réseau.

La première exigence pour la mise en réseau des entreprises est de résoudre la non-interopérabilité des données et des services. HLA fait partie d'une solution. Par exemple dans le cas pratique de la figure 3, l'objectif est d'établir des liens vers un «Bus de Service d'Interopérabilité » (se référant aux concepts de l' «Enterprise Service Bus » de Chapell (2004)), et de connecter de nouvelles fonctionnalités avec des composants déjà connectés (par exemple, DEVS/HLA AnyLogic@, HLA, etc.). Le point suivant détaille comment une plateforme compatible HLA peut faciliter l'intégration de tous les composants.

Le deuxième point consiste à prendre en compte l'exigence d'interopérabilité à chaque étape du processus de développement d'un SI et d'essayer de comprendre comment elle peut faciliter l'interopérabilité à un niveau inférieur d'abstraction. Cette idée introduite dans MDI (Bourey et al., 2007) est de définir des modèles d'interopérabilité aux niveaux des activités de modélisation d'entreprise et de les réutiliser pour faciliter l'interopérabilité des niveaux modélisation de processus, services et données.

\subsection{Plateforme d'interopérabilité de SI basée SMA compatible HLA}

La nouvelle notion d'interopérabilité des entreprises, énoncée dans la section précédente, peut être implémentée au moment de l'exécution par des agents fédérés. Basée sur l'expérience de l'utilisation des SMA/HLA, une mise en œuvre innovante de l'interopérabilité d'entreprise en fédération est proposée (voir figure 5).

Les SI des entreprises sont fréquemment considérés comme des boîtes noires. Ils sont couramment mis au point de façon ad hoc et/ou par un vendeur de solutions. Cela implique qu'aucune action sur son comportement ou modification ne peut être aisément effectuée. De ce fait, notre solution consiste plutôt à ajouter une couche d'interopérabilité entre le SI et son environnement. Précisément, l'idée est d'ajouter des composants pour coder et décoder les informations échangées entre le SI et les applications d'autres entreprises. Cette section dévoile les composants requis pour cette plateforme globale distribuée. La mise en œuvre distribuée implique l'extension de deux composants pour l'entreprise locale afin de définir un «HLA Enterprise Federated Agent»(HEFA). Les éléments de cette nouvelle architecture sont présentés ci-après en rapport avec la description de la figure 5.

Dans l'architecture proposée, les services fournis par les systèmes d'information locaux des entreprises restent inchangés. Des agents logiciels intermédiaires transforment l'information du format local du SI au format commun XML/HLA et 
vice versa. De plus, HLA nécessite l'ajout d'un composant «Local RTI Component » (LRC), qui interface avec les messages d'entrée et de sortie du réseau. L'approche proposée dans cet article ajoute une autre couche composant «Agent Information Coder/Decoder» (AICD), qui est le centre du système Agent. Il s'agit d'un composant logiciel spécifique, nouvellement, introduit dans cette approche. Il interprète les informations reçues, depuis le réseau, pour les transformer vers un format local. Les agents utilisent une ontologie de domaine éphémère (provenant d'une base locale ou de l'extérieur) pour interpréter et transformer l'information. Les agents ont vocation à être implémentés en JAVA sur la plateforme JADE. Ces agents impliqués dans la manipulation ontologique des données sont détaillées ci-après.

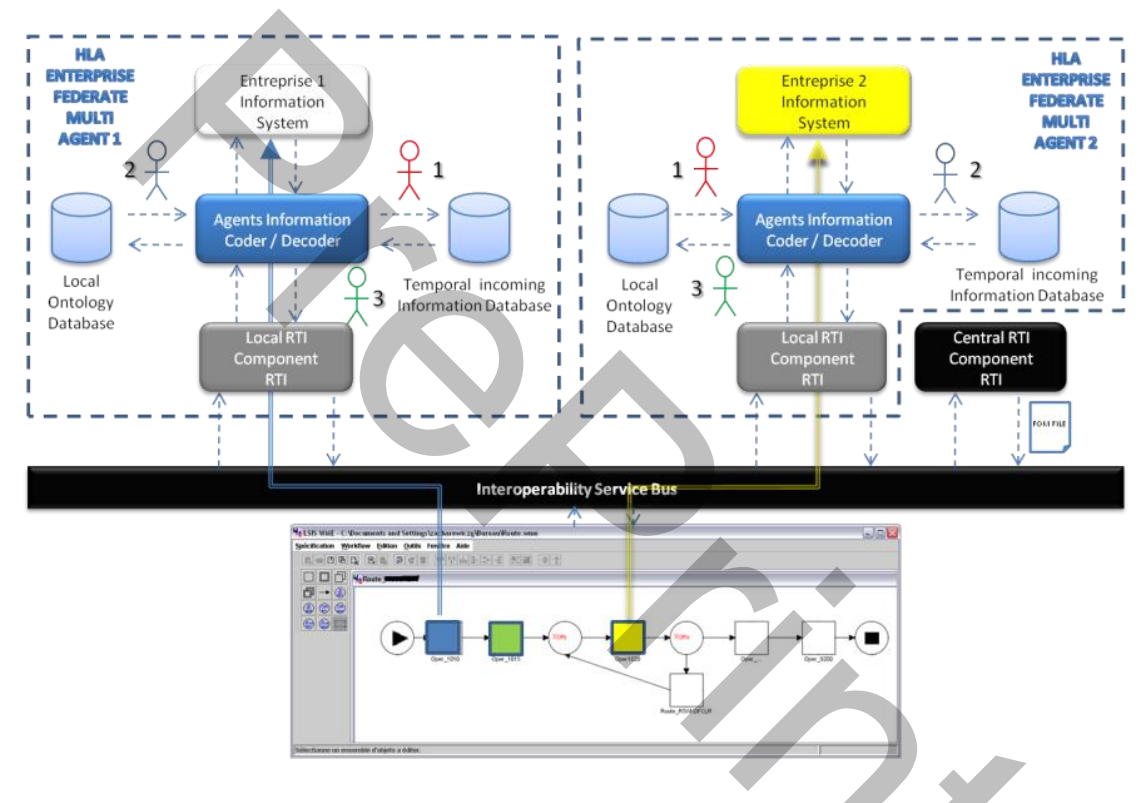

Figure 5. Plateforme d'échange de données par fédération d'agents

L'Agent stockage des données (figure 5 Agent 1) est utilisé pour stocker les informations reçues et vérifier la capacité à décoder l'information en communicant avec l'agent Ontologie. Les informations sont stockées en XML dans une base où les données sont indexées. Une fois l'accord d'utiliser les données reçues, il envoie au système d'information les données au SI. Il a donc également pour charge de transformer les données au format local du SI. Dans le cas pessimiste où le format d'entrée/sortie des SI n'est pas ouvert, les messages seront reconstruits à partir de la déduction des formats, basée sur l'échange de messages tests avec le SI.

L'Agent Ontologie (figure 5 Agent 2) est lié à un référentiel d'ontologies locales, il vérifie la cohérence des informations reçues relatives à l'ontologie locale et décide si les données peuvent être exploitées. La classification d'une donnée (au format 
XML) est réalisée par calcul de proximité avec les concepts présents dans une ontologie OWL (Gandon, 2008). Un pourcentage de vraisemblance est déduit de la présence/absence de termes au niveau de sa structure et des seuils de reconnaissance sont appliqués. Si la donnée est reconnue, il renvoie à l'agent de stockage de données, l'information et l'ontologie à utiliser. Sinon, il demande à l'agent de communication de déclencher un début de dialogue avec l'agent émetteur pour obtenir l'ontologie appropriée. Le scénario de communication utilisé est celui décrit dans le cas b section 3.3 de présentation des ontologies éphémères. Cet agent gère également la validité des ontologies. Il supprime ou déclasse (suppressed or legacy ontology), dans la base locale, les ontologies atteignant leur limite de validité. Notons que des données peuvent parfois être reconnues ou ignorées à tort.

L'Agent Communication (figure 5 Agent 3) reçoit les informations de l'extérieur qu'il transmet à l'agent 1 . S'il est informé de l'incapacité à les utiliser, il va initier une conversation avec l'agent respectif de l'expéditeur des données pour définir les modalités pour recevoir 1'ontologie appropriée. Le dialogue d'Agents est porté par des messages au format du protocole de communication. Dans cette approche, HLA est la solution technique retenue. Le composant est codé en JAVA avec l'utilisation du RTI open source poRTIco (poRTIco, 2010). Le protocole de communication réseau est celui employé par le RTI poRTIco : TCP/IP.

Enfin, la couche «Local RTI Component» (LRC) est le niveau inférieur, c'est le niveau service et données. Il met en œuvre les technologies utilisées pour l'échange d'informations informatisées. Les séquences d'informations à échanger sont orchestrées par un moteur de Workflow visible au bas de la figure 3. Cet outil permet de modéliser la dynamique du processus collaboratif, i.e. la séquence d'information à traiter et l'allocation d'intervention des ressources. Le formalisme graphique retenu est celui des Workflow (Van der Aalst, 2000), (WfMC, 2005). Il fait appel à des éléments descriptifs graphiques : les tâches et les contrôleurs de flux qui dirigent les informations entre les tâches : jonction, disjonction, itération, (voir Zacharewicz (2009)). Cet outil est hébergé sur la plateforme. Dans l'exemple figure 3, on se focalise sur 2 tâches (fléchées sur la figure 5) du processus à exécuter en séquence. Chaque tâche fait appel à un SI d'une entreprise distribuée, et identifie les informations à échanger avec le SI. Dans le flux transitent donc les informations et les ontologies. Le bus est orchestré par HLA sur une base réseau TCP/IP sécurisé.

\section{Bilan d'interopérabilité des plateformes}

Cet article propose une comparaison entre les cinq plateformes HLA, présentées ci-avant, et leurs cartographies dans le cadre d'interopérabilité d'entreprise. Une représentation graphique de la comparaison est illustrée figure 6.

La première plateforme MCDVP (section 4.3.1) est dédiée à l'implémentation technologique de l'échange de connaissances. Elle aborde à presque tous les niveaux d'abstraction les barrières technologiques. En particulier, toutes les barrières sont 
considérées au niveau des données. En contrepartie, les considérations organisationnelles et conceptuelles ne sont pas dans le champ de l'étude. Le problème de l'interopérabilité est principalement exprimé et directement lié aux questions techniques. La vision du niveau décisionnel et stratégique n'est pas exprimée dans la représentation technique.

La deuxième plateforme CEMF-DO (section 4.3.2) est une plateforme dédiée à l'entreprise distribuée. Dans ce cas, le processus est bien décrit dans un modèle. L'entreprise est décrite à l'aide d'UEML (2003) et la synchronisation est assurée entre les modèles de processus. En contrepartie, les rôles des ressources dans l'organisation ne sont pas intégrés au niveau des données. En outre, aucune intégration directe technologique des concepts du niveau business n'est fournie.

La troisième plateforme MISSION (section 4.3.3) est dédiée à la simulation distribuée de la chaîne d'approvisionnement et de production. L'interface utilisateur est générée sur le profil de l'utilisateur en fonction de ses responsabilités. En contrepartie, la modélisation conceptuelle de l'entreprise et de ces processus n'est pas abordée.

La quatrième plateforme LSIS-WME (section 4.3.4) est dédiée à l'interopérabilité des applications dans le contrôle d'une chaîne de production. Elle est centrée sur la modélisation des processus et leurs transformations au niveau des données pour assurer l'exécution du processus. En contrepartie, la modélisation conceptuelle du niveau décisionnel et stratégique de l'entreprise n'est pas abordée.

La cinquième plateforme SMA/HLA (section 5.2) est dédiée à l'interopérabilité des entreprises dans l'échange de documents. Elle est centrée sur la dernière approche en matière d'interopérabilité : l'approche fédérée. Elle utilise les ontologies et les agents logiciels afin d'être agile à toute situation nouvelle, par exemple un nouveau projet, de nouveaux contrats, etc. L'inconvénient est qu'elle n'aborde pas le cahier des charges au niveau des modèles d'entreprises mais commence directement à partir du niveau de modélisation des processus.

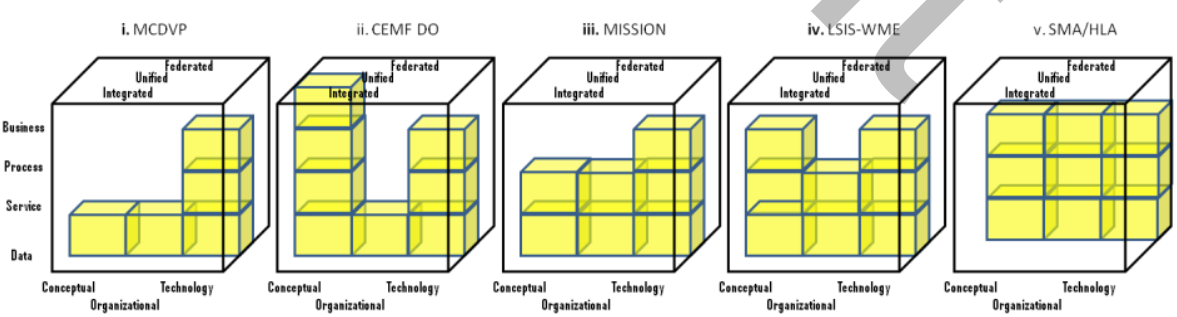

Figure 6. 3D Comparaison des Plateformes d'interopérabilités compatibles HLA 
En résumé, il est possible d'observer que la dimension technologique de l'interopérabilité est bien traitée dans la majorité des plateformes. A l'inverse, les barrières organisationnelles ne sont pas bien identifiées. Certaines plateformes essayent de lutter contre cette barrière au niveau des services et des niveaux supérieurs. En outre, la barrière conceptuelle est principalement abordée à faible niveau d'abstraction (i.e. aux niveaux services et données), mais la plupart des plateformes ne considèrent pas (ou ne documentent pas) un niveau supérieur de spécification (i.e. au niveau processus, stratégique des entreprises). Enfin, la plupart des plateformes collaboratives ayant recouru à HLA sont développées par l'approche unifiée. Les perspectives de solutions par l'approche fédérée sont donc importantes.

Aucune plateforme existante n'est en mesure de résoudre toutes les barrières et couvrir toutes les préoccupations d'interopérabilité en utilisant une approche unique (unifiée, intégrée ou fédérée). Certains domaines d'interopérabilité des entreprises (i.e. certaines briques du cadre d'interopérabilité tridimensionnel, introduit en figure 1) demeurent non solutionnés. L'étude met de plus en évidence que, même si les plateformes sont a priori prétendues collaboratives et interopérables, dans les faits l'effort visant à les rendre inter-interopérables n'est pas trivial. Une méthodologie de développement commune pourrait permettre de réutiliser les plateformes et de comparer plus simplement les différentes approches. La production de spécifications (par exemple des modèles conceptuels) aurait également pu faciliter l'interopérabilité. Ceci rejoint les arguments sur l'intérêt de proposer une méthodologie de développement commune, type MDA, MDI (Zacharewicz, 2009), pour combiner les approches et couvrir plus facilement les obstacles et les préoccupations d'un cas d'interopérabilité (étendre le «mur de brique » du cadre).

\section{Conclusion}

Cet article propose un rappel des concepts d'interopérabilité d'entreprise. Dans le domaine de la mise en interopérabilité des SI d'entreprises, il présente des plateformes d'échanges d'informations entre SI d'entreprises distribuées et hétérogènes, notamment en utilisant la norme HLA. Il est de plus introduit une nouvelle plateforme pour assurer la communication des SI d'entreprises distribuées fortement autonomes. Cette plateforme exécute l'échange d'informations entre entreprises sans ontologie métier commune persistante, en recourant à des ontologies locales éphémères. Concrètement, cette plateforme logicielle permet aux systèmes locaux d'interpréter les informations extérieures par l'utilisation d'une ontologie locale, si elle est présente, ou par le dialogue d'agents logiciels avec d'autres entreprises pour son obtention. Cette ontologie de domaine est éliminée à l'issue d'un projet ou après une durée de validité écoulée. Cette non-persistance de la sémantique des informations renforce la confidentialité et l'agilité des systèmes. Enfin, la plateforme compatible HLA préserve la cohérence dynamique des informations échangées. L'agilité apportée aux SI des entreprises améliore donc leur potentiel d'interopérabilité avec leur environnement. 
Cet article est conclu par une classification des fonctionnalités des plateformes dans le cadre tridimensionnel d'interopérabilité. Les capacités des plateformes sont discutées et font apparaître l'occultation de certains points de vue. Il peut être retenu, en particulier, les absences fréquentes des visions des niveaux décisionnaires ainsi que l'incapacité à transcrire certains concepts métiers et organisationnels des entreprises, dans les solutions développées. La prochaine étape de notre recherche consiste à améliorer la spécification du comportement des agents et la manipulation des ontologies pour augmenter la couverture d'interopérabilité. En outre, un cas réel est en cours d'étude dans le domaine de l'aéronautique. Il consiste en l'échange de données hétérogènes critiques entre sous-traitants et partenaires multiples.

\section{Bibliographie}

Berio G., Mertins K. et Jaekel F.W., "Common Enterprise Modelling Framework for Distributed Organisations", $16^{\text {th }}$ IFAC World Congress, Praha, Elsevier, July 4-8 2005.

Bourey J.P., Grangel Seguer R. et DoumeingtsG., Berre A.J., "Report on Model Driven Interoperability", (INTEROP NoE Contract no.: IST-508011, Delivrable DTG2.3 p. 91), 2007, Retrieved August, 2009. http://interop-vlab.eu/ei_public_deliverables

Chen D., Doumeingts G., "European Initiatives to develop interoperability of enterprise applications - basic concepts, framework and roadmap", Journal of Annual reviews in Control, vol. 27, $\mathrm{n}^{\circ} 2,2003$, p. 151-160.

Chen D., Dassisti M., Elvesæter B., "Enterprise Interoperability Framework and Knowledge Corpus - (INTEROP NoE, Deliverable DI.3-Annex)", May 212007.

Doumeingts G., Méthode GRAI : méthode de conception des systèmes productiques, Thèse de doctorat, Laboratoire d'Automatique et de Productique, Université Bordeaux I, 1984.

Fujimoto R.M., Parallel and Distributed Simulation System, New York, NY, Wiley Interscience, 2000.

Gandon F., Corby, O., Diop I., Lo M. "Distances sémantiques dans des applications de gestion d'information utilisant le web sémantique", Proceedings of the Semantic similarity workshop, EGC, 2008, Sophia-Antipolis, France.

Gruber T., "A translation approach to portable ontology specifications", Knowledge Acquisition, vol. 5, n 2, 1993, p. 199-199.

IDEAS Consortium, “Thematic Network, IDEAS Interoperability Development for Enterprise Application and Software - Roadmaps", (IDEAS T.R. Annex 1), 2002.

IEEE std 1516.2-2000. IEEE Standard for Modeling and Simulation (M\&S) High Level Architecture (HLA) - Federate Interface Specification, New York, NY: Institute of Electrical and Electronic Engineers, 2000.

ISO 14258., Industrial Automation Systems - Concepts and Rules for Enterprise Models (ISO TC184/SC5/WG1), 1999. 
IST. Enterprise Interoperability Research Roadmap (Final Version, Version 4.0), 30 sept. 2007. http://cordis.europa.eu/ist/ict-entnet/ei-roadmap_en.htm.

Jaekel F.W., "Distributed Federated Simulation of Supply Chains" (INTEROP Network of Excellence Contract no. IST-508011, Deliverable D6.1), 2005. http://interop-vlab.eu/ei _public_deliverables

Labarthe O., Espinasse B., Ferrarini A., Montreuil B., "Toward a Methodological Framework for Agent-Based Modelling and Simulation of Supply Chains in a Mass Customization Context", Simulation Modelling Practice and Theory, vol. 15, n² 2, 2007, p. 113-136.

Leigh J., Johnson A. and DeFanti T.A., "CAVERN: A Distributed Architecture for Supporting Scalable Persistence and Interoperability", Collaborative Virtual Environments. Virtual Reality: Research, Development and Applications, vol. 2, $\mathrm{n}^{\circ} 2$, 1997, p. 217-237.

OMG, Object Management group. MDA Guide Version 1.0.1, (Document Number: OMG/2003-06-01), Needham, MA, USA: Object Management Group, 2003.

Panetto H., Zelm M., Scannapieco M., "Interoperability Research for Networked Enterprises Applications and Software", Proceedings of the On the Move to Meaningful Internet Systems 2004 International Workshops, OTM2004, Larnaca, Cyprus, October 272004.

Portico project, http://www.porticoproject.org/.

PSL., Website, 2003, www.mel.nist.gov/psl/

Rabe M. \& Jaekel F.W., "The MISSION Project", Proceedings of the IFIP TC5/WG5.12 International Conference on Enterprise Integration and Modeling Technique, Valencia, Spain, 2002, p. 235-242.

Rada R., Mili H., Bicknell E., Blettner M., "Development and Application of a Metric on Semantic Nets", IEEE Systems, Man, and Cybernetics, vol. 19, n 1, 1989, p. 17-30.

Timmers P., "Business Models for Electronic Markets", Journal on Electronic Markets, vol. $8, \mathrm{n}^{\circ} 2,1998$, p. 3-8.

UEML., Website, 2003, Retrieved October 02, 2009, from http://www.uemlwiki.org/

Ullberg J., Chen D., Johnson P., "Barriers to Enterprise Interoperability, In Enterprise Interoperability”, Business Information Processing, Berlin, Springer, 2009, p. 13-24.

Van Der Aalst W. M. P., Barros Alistair P., Hofstede Arthur H. M. ter, Kiepuszewski Bartek, "Advanced Workflow Patterns", $7^{\text {th }}$ International Conference on Cooperative Information Systems, September 06-08, 2000, p. 18-29.

WfMC, Workflow Management Coalition, Workflow Process Definition Interface - XML Process Definition Language (XPDL), (WFMC-TC-1025), Winchester, Hampshire, United Kingdom: The Workflow Management Coalition, 2005.

Wooldridge M., An Introduction to Multi-agents Systems, New York, NY, Wiley, 2002.

Zacharewicz G., Giambiasi N., Frydman C., "Lookahead Computation in G-DEVS/HLA Environment", Simulation News Europe Journal (SNE) special issue on Parallel and Distributed Simulation Methods and Environments, vol. 16, n 2, 2006, p. 15-24. 
Zacharewicz G., Frydman C., Giambiasi N., "G-DEVS/HLA Environment for Distributed Simulations of Workflows", Simulation, vol. 84, n 5, 2008, p. 197-213.

Zacharewicz G., Labarthe O., Chen D., Vallespir B., "Multi Agent/HLA Enterprise Interoperability (Short-Lived Ontology Based)", $6^{\text {th }}$ Edition of the International Mediterranean Modelling Multiconference, 2009, Puerto de la Cruz, Spain.

Zeigler B.P., Theory of Modeling and Simulation, New York, NY, John Wiley, 1973.

Zhang H., Wang H., Chen D., "A Multidisciplinary Collaborative Design System in a Distributed Environment", $11^{\text {th }}$ Conference on Computer Supported Cooperative Work in Design (CSCWD'07), April 26-28, 2007, Melbourne, Australia, p. 233-239.

Zhong J., Zhu H., Li J., Yu Y., “Conceptual Graph Matching for Semantic Search”, $10^{\text {th }}$ International Conference on Conceptual Structures ICCS 2002, LNCS 2393, Springer Verlag, Borovets, Bulgaria, p. 92-106. 